Virginia Commonwealth University vCU Scholars Compass

\title{
Cation occupancy determination in manganese zinc ferrites using Fourier transform infrared spectroscopy
}

\author{
M. D. Shultz \\ Virginia Commonwealth University \\ E. E. Carpenter \\ Virginia Commonwealth University \\ S.A. Morrison \\ George Washington University \\ S. Calvin \\ Sarah Lawrence College
}

Follow this and additional works at: http://scholarscompass.vcu.edu/chem_pubs

Part of the Chemistry Commons

Shultz, M. D., Carpenter, E. E., Morrison, S. A., et al. Cation occupancy determination in manganese zinc ferrites using Fourier transform infrared spectroscopy. Journal of Applied Physics 99, 08 M901 (2006). Copyright (C) 2006 AIP Publishing LLC.

\section{Downloaded from}

http://scholarscompass.vcu.edu/chem_pubs/40

This Article is brought to you for free and open access by the Dept. of Chemistry at VCU Scholars Compass. It has been accepted for inclusion in Chemistry Publications by an authorized administrator of VCU Scholars Compass. For more information, please contact libcompass@vcu.edu. 


\title{
Cation occupancy determination in manganese zinc ferrites using Fourier transform infrared spectroscopy
}

\author{
M. D. Shultz and E. E. Carpenter \\ Virginia Commonwealth University, Richmond, Virginia 23284 \\ S. A. Morrison ${ }^{\text {a) }}$ \\ George Washington University, Washington, DC 20052 \\ S. Calvin \\ Sarah Lawrence College, Bronxville, New York 10708
}

(Presented on 2 November 2005; published online 17 April 2006)

The magnetic and electric properties of ferrites are influenced by the cation distribution within the crystalline spinel lattice. Methods such as extended x-ray-absorption fine structure (EXAFS) have been used to determine cation occupancies within the crystalline structure of materials such as manganese zinc ferrite (MZFO); however, it is not practical to be used for daily analysis. Fourier transform infrared (FTIR) spectroscopy is another technique which has the potential to determine cation occupancy while offering speed and convenience. In the literature it has been demonstrated that in ferrite systems FTIR data can be correlated to cation percentages when comparing tetrahedral $\left(T_{d}\right)$ and octahedral $\left(O_{h}\right)$ sites. FTIR spectra were collected on a series of MZFO nanoparticles in the range from 200 to $600 \mathrm{~cm}^{-1}$ and two absorbance peaks were observed. The first absorption region shifted with changing sample composition as calculated from transmission EXAFS experiments and elemental analysis. The data was normalized to the maximum of the peak of interest and the shifts were correlated to cation occupancy. () 2006 American Institute of Physics.

[DOI: $10.1063 / 1.2151831]$

This study investigates the use of Fourier transform infrared (FTIR) spectroscopy for determining cation occupancy in manganese zinc ferrite (MZFO). One technique that has been successful in determining cation occupancies within bulk and nanoparticle mixed metal ferrites is extended x-rayabsorption fine structure (EXAFS). ${ }^{1}$ However, this method is not practical for daily laboratory analysis to develop synthetic routes due to the necessity of traveling to a synchrotron-radiation source. It has been demonstrated in ferrite systems that FTIR data can be correlated to cation occupancy in tetrahedral and octahedral sites. ${ }^{2}$ In our work, elemental analysis by inductively coupled plasma-optical emission spectroscopy (ICP) is used to determine the cation concentrations and ratios between the components in the system. Cation distributions within the ferrite lattice, as determined by EXAFS, were then used to establish a correlation with measured FTIR absorption bands. These bands were assigned to different cation-oxygen vibrational frequencies based on the quality of the data fit and trends observed.

To establish the versatility of this method the MZFO data was collected using both nanoparticulate MZFO as well ceramic bulk samples. The ceramic standards were prepared by mixing $\alpha-\mathrm{Fe}_{2} \mathrm{O}_{3}, \mathrm{Mn}_{2} \mathrm{O}_{3}$, and $\mathrm{ZnO}$ powders in a ball mill and subsequent annealing at $1200{ }^{\circ} \mathrm{C} .{ }^{3}$ Reverse micelle techniques were used to synthesize the nanoparticles, as detailed by Calvin et al. ${ }^{1}$ EXAFS spectroscopy was performed at beam line X11A of the National Synchrotron Light Source at Brookhaven National Laboratory. Elemental analyses for the

${ }^{a)}$ Electronic mail: stuu@gwu.edu samples detailed by Calvin et al. are discussed therein; the remaining samples were prepared for ICP in the same manner and analyzed using a Varian Vista-MPX ICP-OES. Samples were prepared for FTIR by grinding them with mortar and pestle into a mixture with silver bromide at approximately a 1:10 mass ratio. The mixture was then pressed in a $13 \mathrm{~mm}$ pellet die at an applied load of 9 tons, to yield approximately 1-mm-thick pellets. The spectra were collected on a Nicolet Nexus 670 FTIR with a polyethylene beam splitter, deuterated triglycine sulfate (DTGS) detector, and a purge cell apparatus to minimize moisture in the measuring environment. A single beam background spectrum was taken to minimize $\mathrm{CO}_{2}$ and $\mathrm{H}_{2} \mathrm{O}$ signals in the measurements. A $\mathrm{AgBr}$ spectrum was collected and used for the background subtraction of each sample. The spectra of the samples were collected in the far-IR region from 600 to $200 \mathrm{~cm}^{-1}$ and normalized to the maximum absorbance value between 450 and $300 \mathrm{~cm}^{-1}$, to account for differences in the thickness and concentration of each pellet.

The FTIR spectra we collected contained two absorption regions between 600 and $200 \mathrm{~cm}^{-1}$. This region was chosen based on a potential-energy study by Waldron in 1955 in which he derived that $480 \mathrm{~cm}^{-1}$ was an approximate mean between the cut-off frequencies of the oxygen and metal cation vibrations. ${ }^{4}$ In our data the first region $\left(\nu_{1}\right)$ did not resolve into separate peaks, while the second region revealed the presence of three bands $\left(\nu_{2}, \nu_{3}\right.$, and $\left.\nu_{4}\right)$, as seen in Fig. 1 . The maximum value of $\nu_{1}$ shifted within the range of 553 and $571 \mathrm{~cm}^{-1}$ for the various samples of MZFO. The percentage of interstitial sites occupied by a specific metal within the spinel structure can be calculated from ICP and 


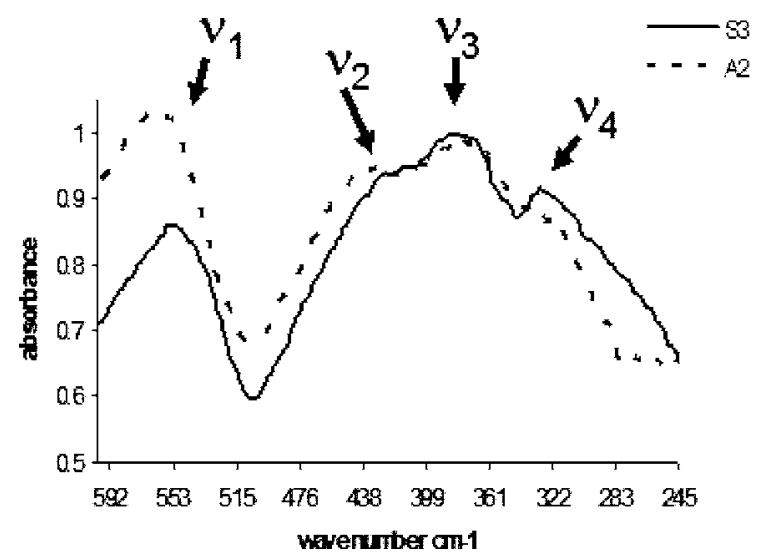

FIG. 1. Plot of absorbance vs wave number for two of the MZFO samples showing all four absorbance bands and the two absorbance regions.

EXAFS data. This allows a direct comparison of the shifts in the absorbance peaks with the percentage of either interstitial site occupied by a given cation.

Two common assumptions are made when investigating MZFO; one is that the zinc occupies only tetrahedral sites, ${ }^{5}$ and the second is that the amount of $\mathrm{Mn}^{3+}$ present is insignificant. Based on these assumptions the percentage of the available octahedral sites occupied by $\mathrm{Mn}^{2+}$ was compared to the changes in the $\nu_{1}$ absorbance band. From this comparison a strong correlation to the EXAFS-determined occupancies was found, as shown in Fig. 2. This plot can also be done with $\mathrm{Fe}^{2+/ 3+}$ octahedral occupancy since the first absorption region is actually a combination of the six coordinated $\mathrm{Mn}^{2+}-\mathrm{O}$ stretch and $\mathrm{Fe}^{2+/ 3+}-\mathrm{O}$ stretch. Since $\mathrm{Mn}^{2+}, \mathrm{Fe}^{2+}$, and $\mathrm{Fe}^{3+}$ have similar masses and similar bond lengths when octahedrally coordinated, ${ }^{5}$ the two stretches appear as one unresolved peak that shifts due to the ratio of concentrations of $\mathrm{Mn}^{2+}$ to $\mathrm{Fe}^{2+/ 3}$.

In the second absorption region, the separate bands do not appear to shift but the relative intensity of each peak changes. This change in intensity of individual bands can be attributed to concentration differences between samples as well as different concentrations of a given metal in tetrahedral sites. The lowest-frequency band, $\nu_{4}$, corresponds to $\mathrm{Zn}^{2+}$, which is the heaviest metal in the system. The change

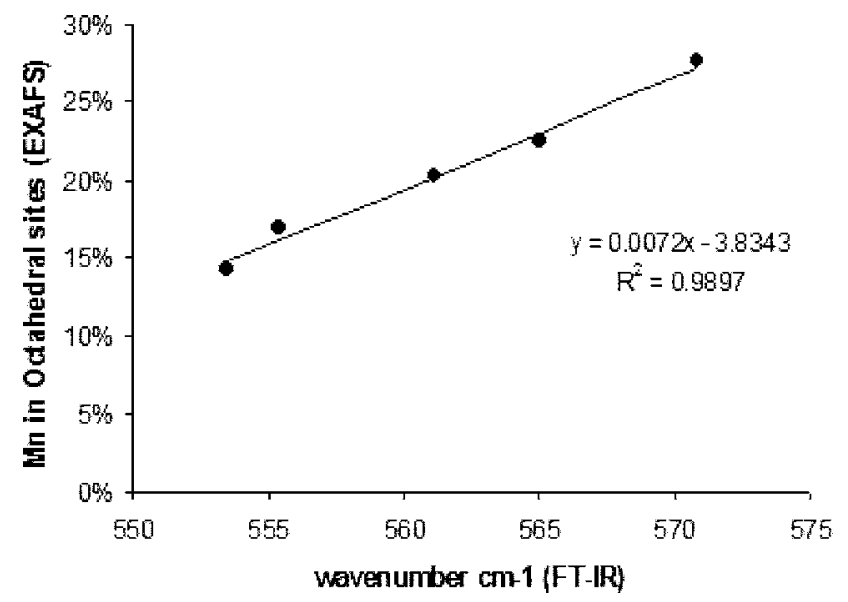

FIG. 2. Plot of the percentage of the available octahedral sites occupied by $\mathrm{Mn}^{2+}$ vs wave number.

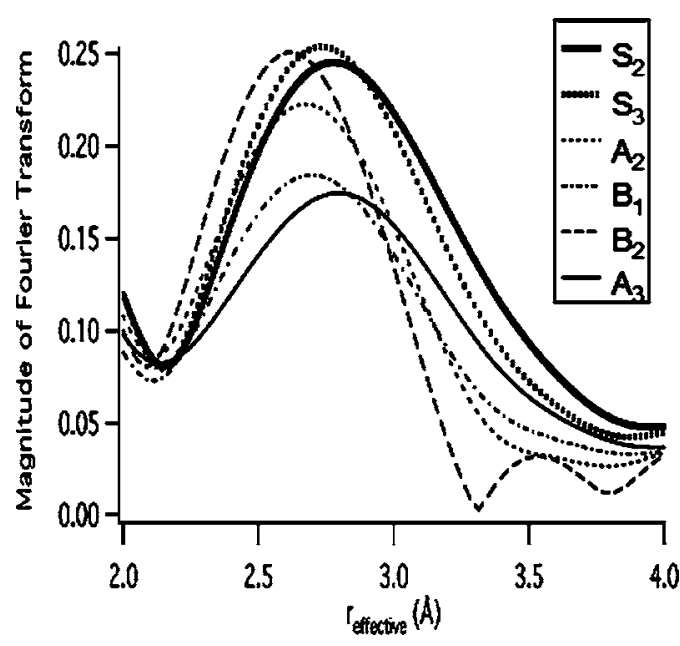

FIG. 3. Plot of the magnitude of Fourier transform vs effective radius ( $k$ weight of 1) for the Mn edge.

in intensity in this band, as seen in Fig. 1, is due to the amount of $\mathrm{Zn}^{2+}$ in the sample. The remaining two absorbance bands therefore correspond to $\mathrm{Mn}^{2+} / \mathrm{Fe}^{2+}-\mathrm{O}$ and $\mathrm{Fe}^{3+}-\mathrm{O}$ when tetrahedrally coordinated. The $\mathrm{Fe}^{3+}-\mathrm{O}$ stretch should have a higher natural frequency than the $\mathrm{Mn}^{2+} / \mathrm{Fe}^{2+}-\mathrm{O}$ stretch due to the significantly shorter bond length of the former. Thus $\nu_{2}$ should correspond to the $\mathrm{Fe}^{3+}-\mathrm{O}$ stretch, while $\nu_{3}$ corresponds to the $\mathrm{Mn}^{2+} / \mathrm{Fe}^{2+}-\mathrm{O}$ stretch. This relationship is verified in our results because the intensity of $\nu_{2}$ increases as the percentage of the tetrahedral sites occupied by $\mathrm{Fe}^{3+}$ increases, allowing the vibrational difference to become more apparent.

These results can be explained in terms of a classical mechanics model in which the cation-oxygen bonds are defined as springs. In comparing the two types of interstitial sites one can envision two harmonic-oscillator systems, each composed of a single mass in the center with one containing four springs and the other containing six springs. In either traverse motion or vibration along an axis, the four-spring system will have a lower natural frequency than that of a six-spring system based on the corresponding equations. For motion along an axis,

$$
\omega \equiv \sqrt{\frac{k}{m}},
$$

and for traverse motion,

$$
\omega \equiv \sqrt{\frac{f}{l_{b}}},
$$

where $\omega$ is frequency, $k$ is the total spring constant of the system, $m$ is mass, $f$ is related to the driving force, and $l_{b}$ is length of the spring which is related to bond length. Therefore, a four-bond system should have a lower natural frequency than the six-bond system given the same central metal.

Qualitative trends in site occupancy can also be seen by looking at the magnitude plots of the Fourier transform in EXAFS analysis. For a $k$ weight of 1 , predominately octahedral occupancy yields a peak at $2.6 \AA$ and a node at $3.3 \AA$, as seen in Fig. 3. High tetrahedral occupancy removes the node 
and shifts the peak toward 3.1 $\AA$. MZFO sample $\mathrm{A}_{3}$ was used as a double-blind validation step in which FTIR and the correlation curve in Fig. 2 were used to predict the site occupancy, which was then compared to independently determined EXAFS results. The two techniques showed impressive agreement, with FTIR indicating that $15 \%$ of the octahedral sites were occupied by manganese, while EXAFS yielded $13+/-2 \%$ for the same value. This is particularly noteworthy because it represents an extrapolation rather than an interpolation; all of the samples used to calibrate Fig. 2 had a greater fraction of octahedral sites occupied by manganese than was found in this sample.

These results show a correlation of FTIR measurements to the data derived from EXAFS analysis. The resulting curve, shown in Fig. 2, can be used to determine the percent- age of the available octahedral sites occupied by $\mathrm{Mn}^{2+}$ and $\mathrm{Fe}^{2+/ 3+}$. Using elemental analysis to determine the total concentration of zinc, manganese, and iron, the percentage of the tetrahedral sites occupied by each can be determined. This demonstrates that FTIR can be used to determine the cation occupancy in mixed metal ferrite systems.

${ }^{1}$ S. Calvin, E. E. Carpenter, B. Ravel, V. G. Harris, and S. A. Morrison, Phys. Rev. B 66, 224405 (2002).

${ }^{2}$ Q. M. Wei, J. B. Li, and Y. J. Chen, J. Mater. Sci. 36, 5115 (2001).

${ }^{3}$ S. A. Morrison, C. L. Cahill, E. E. Carpenter, S. Calvin, and V. G. Harris, J. Appl. Phys. 93, 7489 (2003).

${ }^{4}$ R. D. Waldron, Phys. Rev. 99, 1727 (1955).

${ }^{5}$ J. Smit and H. P. J. Wijn, Ferrites: Physical Properties of Ferrimagnetic Oxides in Relation to Their Technical Applications (Wiley, New York, 1959). 